\title{
Medizin und Ökonomie: Maßnahmen für eine wissenschaftlich begründete, patientenzentrierte und ressourcenbewusste Versorgung. Ein Strategiepapier der Arbeitsgemeinschaft der Wissenschaftlichen Medizinischen Fachgesellschaften (AWMF) Medicine and Economy: Measures for a Science Based, Patient- Centred and Resource Conscious Care. The Association of Scientific Medical Societies' (AWMF) Strategy Paper
}

Autoren

Monika Nothacker ${ }^{1}$, Reinhard Busse ${ }^{2}$, Peter Elsner ${ }^{3}$, Ulrich R. Fölsch ${ }^{4}$, Manfred Gogol ${ }^{5,6}$, Gerhard Jan Jungehülsing ${ }^{7}$, Ina Kopp ${ }^{1}$, Georg Marckmann ${ }^{8}$, Jens Maschmann ${ }^{9}$, Hans-Joachim Meyer ${ }^{5,}{ }^{10}$, Kurt Miller ${ }^{11}$, Wilfried Wagner ${ }^{5,}{ }^{12}$, Albrecht Wienke ${ }^{5,13}$, Klaus-Peter Zimmer ${ }^{14}$, Rolf Kreienberg ${ }^{5}$

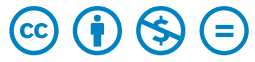

Institute

1 Arbeitsgemeinschaft der Wissenschaftlichen Medizinischen Fachgesellschaften, Institut für Medizinisches Wissensmanagement, c/o PhilippsUniversität Marburg

2 Lehrstuhl für Management im Gesundheitswesen, Technische Universität Berlin, Deutsche Gesellschaft für Gesundheitsökonomie

3 Klinik für Dermatologie und Venerologie, Universitätsklinikum Jena, Deutsche Gesellschaft für Dermatologie

4 Deutsche Gesellschaft für Innere Medizin, Wiesbaden

5 Arbeitsgemeinschaft der Wissenschaftlichen Medizinischen Fachgesellschaften, Berlin

6 Deutsche Gesellschaft für Geriatrie, Deutsche Gesellschaft für Gerontologie und Geriatrie

7 Klinik für Neurologie, Jüdisches Krankenhaus, Berlin; Deutsche Gesellschaft für Neurologie

8 Institut für Ethik, Geschichte und Theorie der Medizin, Ludwigs-Maximilian-Universität München; Akademie für Ethik in der Medizin

9 Universitätsklinikum Jena; Gesellschaft für Qualitätsmanagement im Gesundheitswesen

10 Deutsche Gesellschaft für Chirurgie, Berlin

11 Klinik für Urologie, Charité, Berlin; Deutsche Gesellschaft für Urologie

12 Deutsche Gesellschaft für Mund-, Kiefer- und Gesichtschirurgie

13 Deutsche Gesellschaft für Medizinrecht

14 Allgemeine Pädiatrie und Neonatologie, Universitätsklinikum Gießen; Deutsche Gesellschaft für Kinder- und Jugendmedizin
Schlüsselwörter

Gesundheitsversorgung, Ökonomisierung, Evidenzbasierung, Patientenzentrierung, bedarfsorientierte Planung

Key words

health care, economization, evidence-based medicine, patient-centring, demand responsive planning

Bibliografie

DOI https://doi.org/10.1055/a-0891-3940

Online-Publikation: 16.5.2019

Dtsch Med Wochenschr 2019; 144: 990-996

(C) Georg Thieme Verlag KG, Stuttgart · New York

ISSN 0012-0472

Korrespondenzadresse

Dr. med. Monika Nothacker, MPH

AWMF-Institut für Medizinisches Wissensmanagement,

c/o Philipps-Universität Marburg, Karl-von-Frisch-Str. 1,

35043 Marburg

nothacker@awmf.org

\section{ZUSAMMENFASSUNG}

Die AWMF und ihre Fachgesellschaften nehmen eine zunehmende Dominanz betriebswirtschaftlicher Ziele im stationären Gesundheitssektor wahr, die sich negativ auf die Patientenversorgung auswirken. Während eine angemessene, effiziente und gerechte Mittelverwendung geboten ist, belastet „Ökonomisierung“ Ärzte und Pflegende sowie die weiteren Gesundheitsberufe.

Die AWMF hat zusammen mit Fachgesellschaftsvertretern Ursachen analysiert und Maßnahmen für eine wissenschaftliche, patientenzentrierte und ressourcenbewusste Medizin erarbeitet. Es bestehen Fehlanreize durch Vergütung, Zahl und Ausstattung von Krankenhäusern bzw. Fachabteilungen und deren Grundfinanzierung. Maßnahmen werden für die Patient-ArztEbene, das Krankenhausmanagement, die Planung sowie Finanzierung von Krankenhäusern einschließlich der Vergütung 
stationärer Leistungen vorgeschlagen. Dabei bedarf es der gemeinsamen Anstrengung aller Akteure, um den Patienten und seine Gesundheit wieder in den Mittelpunkt zu stellen.

\section{ABSTRACT}

The AWMF and its medical societies perceive an increasing dominance of economic targets in the hospital health care sector, leading to impairment of patient care. While resource use in health care should be appropriate, efficient and fairly allocated, "economization" creates a burdensome situation for physicians, nurses and other health care professionals.
The AMWF and the medical societies studied causes and developed measures for a scientific, patient-centred and resourceconscious medical care. Disincentives due to the remuneration system, number and equipment of hospitals resp. specialist departments and their basic funding need to be overcome. Proposed actions relate to the patient-doctor-level, the management level of hospitals and the level of planning and financing hospitals including compensation of hospital care. To place patients and their health in the forefront again, joint efforts of all stakeholders in health care are needed.

\section{Anliegen und Ziel}

Die AWMF und ihre Fachgesellschaften treten für eine wissenschaftlich begründete, patientenzentrierte Medizin ein [1]. Sowohl einer Unter-, Über- als auch einer Fehlversorgung ist entgegenzutreten [2]. Im solidarisch finanzierten Gesundheitssystem ist zudem eine angemessene, effiziente und gerechte Verwendung der zur Verfügung gestellten Mittel geboten. Insofern sind Medizin und Ökonomie nicht zu trennen.

Ökonomische Interessen dürfen jedoch medizinische Entscheidungen nicht unangemessen beeinflussen [3, 4]. Nach der Berufsordnung (§2 Abs. 4) dürfen Ärzte hinsichtlich ihrer ärztlichen Entscheidungen keine Weisungen von Nicht-Ärzten entgegennehmen. Unter den jetzigen Rahmenbedingungen kommt es allerdings zunehmend zu Konflikten zwischen betriebswirtschaftlichen Anforderungen und einer evidenzbasierten, patientenzentrierten Versorgung [5-11].

Die daraus resultierende „Ökonomisierung“ - eine Entwicklung, bei der betriebswirtschaftliche Erwägungen jenseits ihrer Dienstfunktion zunehmende Definitionsmacht über individuelle und institutionelle Handlungsziele in der Patientenversorgung gewinnen [4] - gefährdet die an den Bedürfnissen von Patientinnen und Patienten orientierte, evidenzbasierte Medizin, das Patientenwohl und die Versorgungsgerechtigkeit. Zudem belastet sie Ärztinnen und Ärzte, Pflegende sowie andere Berufsgruppen im Gesundheitswesen [9] ${ }^{1}$. Die AWMF verfolgt diese Entwicklung mit großer Sorge.

Die Mitglieder der Wissenschaftlichen Medizinischen Fachgesellschaften sind als klinisch und wissenschaftlich Tätige Gestaltende des Gesundheitswesens. Sie entwickeln interdisziplinäre, multiprofessionelle, evidenzbasierte Leitlinien für die medizinische Versorgung und Empfehlungen für die Sicherstellung notwendiger Strukturen. Sie gestalten deren Umsetzung in freiwilligen Initiativen des Qualitätsmanagements und der Qualitätsverbesserung, z. B. durch Zertifizierungsverfahren, professionelles Peer-Review, Benchmarking und Register. Sie beraten und unterstützen mit ihrer fachlichen Expertise die Institutionen des Gesundheitswesens in wissenschaftlichen Beiräten und Kuratorien. Sie setzen sich in der individuellen Versorgung auf der Patient-Arzt-Ebene für die wissen-

1 Im Folgenden wird zur besseren Lesbarkeit nur von „Patienten“ und „Ärzten“ gesprochen. schaftlich begründete, partizipative Entscheidungsfindung und den vernünftigen Umgang mit den im Gesundheitssystem begrenzt verfügbaren Ressourcen ein. Die Wissenschaftlichen Medizinischen Fachgesellschaften in der AWMF sehen sich jedoch auch in der Verantwortung, kritische Entwicklungen der Gesundheitsversorgung zu adressieren und der Politik Lösungsvorschläge im Sinne konkreter Maßnahmen zu unterbreiten.

Der Fokus gilt im ersten Schritt vornehmlich dem Krankenhaussektor, da sich dort die Konflikte zwischen betriebswirtschaftlichen Zielen und medizinisch-ethischen Anforderungen in besonderer Schärfe manifestieren. Die AWMF ist sich bewusst, dass Lösungen über die Sektorengrenzen hinweg gedacht und implementiert werden müssen. Alle Entscheidungsträger und Akteure sind aufgefordert, in ihrem jeweiligen Handlungsfeld die erforderlichen Schritte zur Versorgungsverbesserung anzugehen. Diese Schritte bedürfen einer gemeinsamen Abstimmung. Die AWMF sucht den konstruktiven Dialog mit allen beteiligten Akteuren in Politik, Management, Selbstverwaltung und Patientenvertretungen.

\section{Maßnahmen}

\section{Patienteninformation und partizipative Entschei- dungsfindung}

Die Wissenschaftlichen Medizinischen Fachgesellschaften sehen Ärzte in der Verantwortung, bedarfsgerechte und ressourcenbewusste Entscheidungen aufgrund einer wissenschaftlich begründeten, individuell abgestimmten Indikationsstellung zu treffen. Dazu bedarf es der gemeinsamen Entscheidungsfindung von Patient und Arzt und einer ganzheitlichen Betreuung von Patienten und Angehörigen.

Empfehlungen für eine sachgerechte Versorgung im Sinne einer wissenschaftlich basierten Medizin sollen Ärzten sowie Pflegenden und Angehörigen weiterer Gesundheitsberufe, aber auch Patienten in geeigneter, leicht zugänglicher Form zur Unterstützung einer partizipativen Entscheidungsfindung für eine patientenorientierte Versorgung als Leitlinien zur Verfügung stehen. Die AWMF und ihre Fachgesellschaften empfehlen regelmäßige fachspezifische und Team-Fortbildungen, um zu verstehen, wie Leitlinienempfehlungen in Behandlungspfade etc. als Entscheidungskorridore unter Einbeziehung von Patientenpräferenzen zu 
integrieren sind. Der adäquaten Indikationsstellung ist ein besonderer Stellenwert einzuräumen.

Für Leistungen, für die besonders häufig Über-, Fehl- oder Unterversorgung festzustellen sind, sollten „Gemeinsam Klug Entscheiden“-Empfehlungen einschließlich Patienteninformationen aus Leitlinien entwickelt und eingesetzt werden [2, 12]. Dabei sind digitale Formate zu fördern, die am unmittelbaren Ort der Behandlung („Point of care“) zur Anwendung kommen, idealerweise mit der Möglichkeit eines direkten Abgleichs von Patientendaten [13]. Bei relativer oder absoluter Mangelausstattung - bezogen auf in Leitlinien konsentierte Behandlungsstandards - müssen potenzielle Patienten im Sinne der „Einrichtungsaufklärung" entsprechend informiert werden [14].

Für ein ganzheitlich ausgerichtetes Patient-Arzt-Verhältnis ist ausreichend Zeit im Arbeitsalltag vorzusehen [15]. Dies beinhaltet eine umfassende Anamnese ebenso wie die Besprechung der verschiedenen möglichen Maßnahmen und Zeit für die gemeinsame Entscheidungsfindung, ggf. unter Einbeziehung von Angehörigen oder anderen Bezugspersonen. Des Weiteren muss genügend Zeit eingeräumt werden für interdisziplinäre bzw. interprofessionelle Fallkonferenzen und Abstimmungen.

\section{Führungsverantwortung und Management im Krankenhaus}

Managemententscheidungen im Krankenhaus müssen sich vorrangig an qualitativ hochwertiger Patientenversorgung im Sinne des Versorgungsauftrags orientieren („ressourcenbewusste Daseinsfürsorge“) statt sich nur an betriebswirtschaftlichen Zielgrößen (z. B. Erlössteigerung) auszurichten. Dazu ist eine gemeinsame Krankenhausführung - Ärztliche Direktion, Pflegedirektion, kaufmännische Leitung - mit Verhandlungen auf Augenhöhe erforderlich [16]. Die Personalausstattung und die Arbeitsbedingungen von Pflegenden, Ärzten und anderen Gesundheitsberufen müssen eine evidenzbasierte, patientenzentrierte Versorgung ermöglichen.

Management- und Führungskonzepte sollten medizinische und wirtschaftliche Erwägungen integriert berücksichtigen, statt diese in Opposition zu stellen [17]. Klinisch tätige Ärzte (v.a. leitende Ärzte) sind in übergeordnete Managementaufgaben im Krankenhaus adäquat einzubinden, im Sinne eines kontinuierlichen Dialogs zwischen Klinikern und Geschäftsführern und als Entscheidungsträger in ihren medizinisch-fachlichen Verantwortungsbereichen einschließlich des Qualitätsmanagements. Die ärztliche Direktion sollte von den Abteilungsleitern vorgeschlagen werden, ggf. als ärztliches Team aus Klinikdirektoren bei nebenamtlicher Funktion. Als zielführend wird - zusammen mit einer kontinuierlichen Prozessoptimierung - die Verwirklichung eines patienten- und personalwertschätzenden Krankenhausmanagements bzw. eines Wertemanagements mit Berücksichtigung ethischer Grundsätze im Sinne des Patientenwohls und der evidenzbasierten Medizin gesehen [17]. In dieses sollten Angehörige aller Gesundheitsberufe einbezogen werden. Wir schlagen vor, diese Aspekte künftig in Qualitätssicherungsverfahren obligat zu berücksichtigen.
Evidenz- und konsensbasierte Leitlinien sollen zur fachlichen Orientierung in den Qualitätsmanagementsystemen der Kliniken hinterlegt werden.

Die Abschaffung fallzahlbezogener/bonusabhängiger Chefarztverträge, die seit langem gefordert wird, ist unbedingt durchzusetzen, ggf. auch mit weiteren gesetzlichen Nachbesserungen, hier gibt es weiterhin deutlichen Handlungsbedarf $[11,18]$. Werden Aufgaben wie Infektionsschutz oder Arbeitsschutz in Chefarztverträgen benannt, sind auch entsprechende personelle und sachliche Ressourcen aufzuführen. Leitende Ärzte sollen weiterhin aktiv auf Missstände aufmerksam machen können (z. B. wenn das Arbeitszeitgesetz aufgrund von Mitarbeitermangel nicht eingehalten werden kann). Der ärztlichen und pflegerischen Leitung muss in begründeten Fällen ein fachliches „Vetorecht“ bei Fragen der Versorgungsqualität bzw. Patientensicherheit zugestanden werden. Eine adäquate Personalpolitik mit entsprechender Ausstattung ist ebenfalls in Qualitätsmanagement-Vorgaben aufzunehmen. Diese darf nicht konterkariert werden, indem Erlöse den Abteilungen „abgezogen“ werden. Die Erlöse (aus den Fallpauschalen - Diagnosis-Related-Groups - DRG) sollen für die Zwecke zur Verfügung stehen, für die sie vorgesehen sind: Zur Deckung der laufenden Personal- und Sachkosten und nicht für Investitionen oder abgeschöpften Gewinn.

Die AWMF fordert Ärzte dazu auf, den Interessenkonflikt zwischen evidenzbasierter Medizin und Patientenorientierung einerseits und den bestehenden Anreizen zur Leistungsausweitung und damit potenzieller Über-, Fehl- bzw. Unterversorgung andererseits offen und explizit zu thematisieren.

\section{Krankenhausplanung und Finanzierung von Kranken- häusern sowie Vergütung stationärer Leistungen}

Die Krankenhausplanung muss im Rahmen regionaler, sektorenübergreifender Versorgungskonzepte bedarfsorientiert gestaltet werden, mit dem Ziel, stationäre Überkapazitäten zu reduzieren. Darüber hinaus ist eine Weiterentwicklung des Vergütungssystems zum Abbau von Fehlanreizen dringend geboten.

Der Abbau der Dysbalance von Überkapazitäten im stationären Sektor einerseits und dem Fehlen von Primärärzten, z. T. auch Fachärzten, andererseits, erfordert eine bedarfsorientierte, sektorenübergreifende Planung mit regionaler Ausrichtung. Die ambulanten und teilstationären Strukturen müssen einbezogen und entsprechend angepasst werden. Die Planung muss fächerspezifisch bzw. ggf. fachabteilungsspezifisch erfolgen, da zwischen einzelnen Fachgruppen aufgrund der Fallpauschalen-bezogenen Anreize für Kliniken große Versorgungsunterschiede bestehen. Für eine solche Planung sind Instrumente zur Bedarfsanalyse für die künftige stationäre Versorgung zu entwickeln, die die demografische Entwicklung und vorhandene Bedingungen (z. B. StadtLand- oder regionales Gefälle) berücksichtigen [19, 20]. Es müssen klare Anforderungen an eine qualitativ hochwertige Versorgung und deren adäquate Refinanzierung festgelegt werden. Zu diskutieren sind gesetzliche Festlegungen auf Landes- und Bundesebene. Die Krankenhausplanung im Rahmen regionaler Versorgungskonzepte bedarf der Unterstützung aller Akteure und Betroffenen - auch bei ggf. schmerzhaften Einschnitten. Eine zentralisiertere Versorgung ist nicht als Widerspruch zu, son- 
dern als Förderung einer qualitativ hochwertigen flächendeckenden Versorgung zu entwickeln.

Fehlanreize im DRG-System, die u. a. zu unangemessenen Leistungsausweitungen und „Portfolioanpassungen“ führen, müssen dringend eliminiert oder zumindest reduziert werden. Die Vergütung sollte sich dabei an einem Modell patientenzentrierter Medizin orientieren [21]. Eine solche Anpassung muss z. B. die Anreize für stationäre Aufnahmen aus den Notfallaufnahmen deutlich reduzieren, z. B. durch eine nicht diagnosebezogene Vergütung einer lediglichen Beobachtung. Sie bedeutet aber auch die angemessene Berücksichtigung der Vergütung von Kommunikation und Indikationsstellung sowie der interdisziplinären Betreuung. Zuschläge für die Betreuung besonderer Patientengruppen sind zu diskutieren. Dabei sind insbesondere Krankenhäuser der Maximalversorgung und Universitätskliniken adäquat zu berücksichtigen, aber auch die Spezial- und transsektorale Versorgung.

Die Wissenschaftlichen Medizinischen Fachgesellschaften sind bereit, sich aktiv in diesen Prozess einzubringen, indem sie die jährlichen Anpassungen der Operations- und Prozedurenschlüssel (OPS) und die Internationale statistische Klassifikation der Krankheiten (ICD - German Modification) beim Deutschen Institut für Medizinische Dokumentation und Information (DIMDI) mitgestalten, z. B. anhand nach Priorisierungskriterien ausgewählter Leitlinienthemen. Die AWMF kann die Abstimmung und Bündelung von Vorschlägen unterstützen.

Zur Verbesserung qualitätsfördernder Strukturen, z. B. durch Förderung von Zertifizierungsinitiativen, bieten die Wissenschaftlichen Medizinischen Fachgesellschaften ihre Zusammenarbeit mit dem Gemeinsamen Bundesausschuss und dem Institut für Qualität und Transparenz im Gesundheitswesen an, um von den Fachgesellschaften entwickelte fachlich-strukturelle Voraussetzungen einzubringen.

Die Wissenschaftlichen Medizinischen Fachgesellschaften fordern darüber hinaus eine angemessene Investitionsfinanzierung für den bedarfsgerecht umgestalteten stationären Sektor. Diese Forderung adressiert bei Beibehaltung der dualen Finanzierung die Länder, bei Übernahme eines Modells wie vom Sachverständigenrat 2018 vorgeschlagen auch den Bund (stärkere finanzielle Beteiligung des Bundes bei Erhalt der Entscheidungshoheit der Länder, s. Abschnitt Krankenhausfinanzierung). Privat geführte Krankenhäuser sollen ausschließlich im Sinne von Non-ProfitUnternehmen geführt werden. Die Erlösentnahme aus Beiträgen der Patientenversorgung (DRGs) zur Generierung von Profiten wird nachdrücklich abgelehnt.

Die Wissenschaftlichen Medizinischen Fachgesellschaften schlagen vor, ein gemeinsames Diskussionsforum mit Vertretern der Bundes- und Landespolitik, der ärztlichen Selbstverwaltung und Patientenorganisationen zum Sicherstellungsauftrag und dessen patientenorientierter Umsetzung in den einzelnen Bundesländern einzurichten.

\section{Hintergrundinformationen}

Änderungen im Krankheitsspektrum, Entwicklungen
in Diagnostik und Therapie, ökonomische Folgen

Die deutsche Bevölkerung wird anteilmäßig älter, was eine Zunahme von altersassoziierten Erkrankungen bedingt, die andere, aber nicht notwendigerweise deutlich mehr medizinische Leistungen benötigen $[22,23]$. Auf der anderen Seite beinhaltet die wieder ansteigende Geburtenrate besondere Herausforderungen (z. B. der Prävention), da das erreichbare Höchstmaß an Gesundheit von Kindern nach der Kinderrechtskonvention vorrangig zu berücksichtigen ist [24]. Viele Erkrankungen sind besser behandelbar als früher, es werden längere Überlebenszeiten erreicht, dies gilt auch für angeborene und/oder seltene Erkrankungen bzw. Erkrankungen des Kindes- und Jugendalters [25]. Diese Behandlungen sind oft kostenintensiv.

Das Krankheitsspektrum verschiebt sich weiterhin hin zu mehr chronischen Erkrankungen und psychischen/psychosomatischen Erkrankungen mit einer steigenden Zahl an multimorbiden Patienten [26, 27]. Dies erfordert zunehmend langfristige Behandlungen und umfassende Betreuung, mit einem hohen Bedarf an Vernetzung. Der ganzheitliche Blick wird allerdings während der ärztlichen Aus-, Weiter- und Fortbildung erschwert, da diese in einer zunehmenden Spezialisierung und Fächer-Aufsplittung stattfindet [28]. Die Anforderungen an diese komplexe Behandlungs- und Betreuungssituationen sind in den Fallpauschalen (DRG) derzeit nicht adäquat abgebildet. Dagegen ist im europäischen Vergleich eine Häufung und Zunahme einzelner Elektiveingriffe zu verzeichnen [29].

Die Einnahmebasis der umlagefinanzierten GKV wird längerfristig durch den steigenden Anteil nicht mehr Erwerbstätiger/ Rentner geschwächt, insbesondere ab Renteneintritt der geburtenstarken Jahrgänge („Babyboomer“) [30]. Dies erfordert einen sehr bewussten Umgang mit knapper werdenden Ressourcen.

\section{Zugang zu und Angebot von stationären Gesundheits- einrichtungen}

Der Zugang zu stationären Einrichtungen ist in Deutschland nicht begrenzt, die Zahl stationärer Einrichtungen, Betten und Fallzahlen liegt deutlich über EU- bzw. OECD-Durchschnitt [31]. Deutschland zeichnet sich durch viele kleine Krankenhäuser aus, deren Ausstattung häufig für eine qualitativ hochwertige Versorgung nicht ausreichend ist. Vielfach sind deshalb Verlegungen erforderlich und häufig bestehen nur kleine Fallzahlen für bestimmte Eingriffe [32]. Dabei gibt es Assoziationen von niedrigen Fallzahlen mit höheren Komplikationsraten und höherer Mortalität für viele Indikationen [33].

Für bestimmte Erkrankungen findet eine Verlagerung von Diagnostik und Therapie in den ambulanten Bereich statt (z. B. onkologische Therapien, chronisch entzündliche Darmerkrankungen oder Rheuma). Geplante stationäre Einweisungen nehmen insgesamt zwar ab, aber Notaufnahmen entwickeln sich zum zentralen Ort für Krankenhausaufnahmen [34]. Hierfür können häufig auch abrechnungsbedingte Gründe angenommen werden, da die Vergütung ambulanter Leistungen für Krankenhäuser, insbesondere Universitätskliniken, nicht kostendeckend ist und stationäre Aufnahmen deutlich attraktiver sind (pers. Mitteilungen).

\section{Rahmenbedingungen stationärer Versorgung}

Die sektorale Gliederung des deutschen Gesundheitssystems in ambulante und stationäre Versorgung und Rehabilitation bedingt jeweils getrennte Akteure, Regulierungen, Finanzierungstöpfe 
und -modalitäten, was einer sektorübergreifenden „integrierten“ Versorgung bzw. der jeweils sinnvollsten Leistungserbringung abträglich ist. Im SVR-Gutachten 2018 wird deshalb eine sektorenübergreifende Versorgungsplanung vorgeschlagen [12].

Ebenfalls abrechnungsbedingte verkürzte Liegezeiten der Patienten bedingen bei wenig sinkender Bettenzahl einen höheren Turnover [35], im Zusammenspiel mit geänderten Arbeitszeiten für ÄrztInnen (Arbeitszeitgesetz) und mehr Teilzeitstellen, fehlstrukturiertem Management sowie einem drastischen Mangel an Pflegekräften [36] führt dies vielfach zu einem angespannten Arbeitsklima inkl. Personalfluktuation und Krankenstand [37-41]. Dies erhöht das Gefährdungsrisiko (Komplikationen, Sterblichkeit) für Patienten. Im stationären Sektor besteht seit 2004 eine „leistungsorientierte“ Vergütung von Fällen, klassifiziert durch Diagnosis-Related-Groups [42] mit bundesweiten Relativgewichten und fast identischem Basisfallwert $[42,44]$, erarbeitet durch ein Institut zur DRG-Weiterentwicklung [45].

\section{Fehlentwicklung unterstützt durch Vergütungssystem und Gewinnorientierung}

Das DRG-Vergütungssystem erfordert eine prospektive Leistungsplanung mit Angaben von Fallzahlen für das nächste Jahr (Erlösbudget). Da reine Vorhaltekosten der Krankenhäuser - Löhne und Gehälter des Personals, Energie, Infrastruktur etc. - nicht adäquat berücksichtigt sind, besteht ein Anreiz zu Fallzahlerhöhung und Aufsplittung von komplexen Fällen zu besser vergüteten Einzelfällen.

Das DRG-System vergütet Krankenhäusern die bei der Versorgung durchschnittlich entstehenden Kosten, wobei bestimmte Leistungen (insbesondere operative Eingriffe, aber z. B. auch Beatmungen) für eine DRG-Fallgruppe konstitutiv sind, während andere Leistungsbestandteile nicht Voraussetzung für die Abrechnung einer DRG sind. So ist die sprechende Medizin im Sinne der gemeinsamen Entscheidungsfindung mit Patienten bzw. die Betreuung unter Einbeziehung der Angehörigen (inkl. Eltern, Sorgeberechtigte) im Verlauf der zeit- und personalintensiven Behandlung von z. B. Kindern und Jugendlichen, aber auch von gebrechlichen Älteren, für die Vergütung nicht konstitutiv [16]. Auch die interdisziplinäre bzw. interprofessionelle Abstimmung [15] sind so weitgehend „unvergütet“, ebenso wie Leistungen für Bedürfnisse von besonderen Patientengruppen (z. B. bei der Transition).

Dagegen nehmen Beteiligte deutliche Anreize für Leistungsänderungen von Krankenhäusern wahr zugunsten lukrativerer Leistungen („Portfolioänderungen“, „Rosinenpicken“) mit zum Teil fraglicher Indikationsstellung [7]. Dies hat zur Konsequenz, dass schlechter vergütete Leistungen ggf. nicht mehr erbracht werden und der Fokus auf das Erreichen hoher Fallzahlen gut vergüteter Leistungen gelegt wird [9]. Es ist zu befürchten, dass diese Entwicklung zulasten finanziell unattraktiver Patientengruppen weiter fortschreitet.

Seit mehr als einem Jahrzehnt sind diese Fehlanreize in der Vergütung der Krankenhausleistungen bekannt, wurden bisher jedoch nicht durch eine Weiterentwicklung des Vergütungssystems korrigiert [46]. Die im SGB V verankerte Grundmaxime nach einnahmeorientierten Ausgaben wird insbesondere im stationären Sektor verletzt; während die einzelnen Fälle im „Preis“ real konstant vergütet werden, führen kontinuierlich steigende stationäre Fallzahlen zu höheren Krankenhausausgaben [47].

Die Investitions-Finanzierung im stationären Bereich ist in hohem Maße unzureichend. Die für die Krankenhausplanung und -ausstattung zuständigen Länder [43] haben einen Investitionsstau zu verantworten, der die oft mangelnde Prozess-Effizienz inkl. fehlender Umsetzung von Digitalisierungskonzepten in vielen Krankenhäusern verstärkt $[48,49]$ und eine systemwidrige Querfinanzierung aus DRG-Erlösen zwingend erforderlich macht $[9,50]$, was strikt abzulehnen ist.

Gleichzeitig wurde die Privatisierung von Krankenhäusern mit gewinnorientierten Konzernstrukturen akzeptiert [51, 52] und damit die profitbestimmte Erbringung von Gesundheitsdienstleistungen (2017: 36,5\% aller Krankenhäuser mit 17,8\% der aufgestellten Betten) bei bestehenden Überkapazitäten.

\section{Qualitätserfassung lückenhaft}

Zur Weiterentwicklung der Finanzstruktur und der Qualität in der gesetzlichen Krankenversicherung wurde im Krankenhausstrukturgesetz [53] die Beauftragung des Instituts für Qualität und Transparenz im Gesundheitswesen (IQTIG) mit Start 2015 gesetzlich festgeschrieben [54]. Teil des gesetzlichen Institutsauftrags ist die Entwicklung planungsrelevanter Indikatoren sowie „qualitätsorientierter“ Zu- und Abschläge, die durch den Gemeinsamen Bundesausschuss (G-BA) in Richtlinien umgesetzt werden [55]. Das gesetzlich vorgeschriebene Qualitätsmanagement und die verpflichtenden Qualitätsindikatoren sind jedoch bisher ungeeignet, die Qualität der gesamten Versorgung bzw. des Gesundheitssystems zu messen und transparent zu machen, da sie auf kleine Teilbereiche bezogen sind [56].

\section{Fazit}

Die AWMF und ihre Fachgesellschaften nehmen eine zunehmende Dominanz betriebswirtschaftlicher Ziele vor allem im stationären Gesundheitssektor wahr, die sich negativ auf die Patientenversorgung auswirken und diese gefährden. Es bestehen Fehlanreize gegen eine patientenorientierte, wissenschaftliche Medizin durch das Vergütungssystem, die Anzahl und Ausstattung von Krankenhäusern bzw. Fachabteilungen und deren Grundfinanzierung.

Um dieser Entwicklung entgegenzuwirken, werden Maßnahmen auf allen Ebenen des Gesundheitssystems vorgeschlagen. Es bedarf der gemeinsamen Anstrengung aller Akteure, der „Ökonomisierung“ in der Medizin entgegenzuwirken und den Patienten und seine Gesundheit wieder in den Mittelpunkt zu stellen.

Auf der Ebene Patient-Arzt ist der Ausbau der sprechenden Medizin adressiert, eine Stärkung der gemeinsamen Entscheidungsfindung und der interdisziplinären Abstimmung. Durch die konsequente Implementierung von Leitlinien und von laienverständlichen Formaten („Gemeinsam Klug Entscheiden“) sollen mögliche Überdiagnostik und Übertherapie klar adressiert werden, aber auch Fehlentwicklungen im Sinne einer Unterversorgung.

Krankenhausleitungen haben den Auftrag, Wertemanagement- und Führungskonzepte zu verwirklichen, die medizinische und wirtschaftliche Erwägungen gleichermaßen berücksichtigen, anstatt sich vorrangig an betriebswirtschaftlichen Anforderungen auszurichten. Dafür bedarf es einer gemeinsamen Führung (Ärzt- 
liche Direktion, Pflegedirektion, kaufmännische Leitung). Die Vergütung nach Fallpauschalen ist im Sinne einer patientenorientierten Medizin anzupassen, Fehlanreize für Interventionen sind zu korrigieren. Die „sprechende Medizin“ einschließlich der interdisziplinären Abstimmung im Krankenhaus und mit weiteren Kollegen sollte besser vergütet werden.

Der Krankenhaussektor insgesamt kann nicht weiter isoliert betrachtet werden. Die Planung muss nach Bedarf erfolgen im Rahmen sektorübergreifender Konzepte. Dabei sind vorhandene stationäre Überkapazitäten abzubauen und geeignete teilstationäre und ambulante Strukturen zu entwickeln und vorzuhalten. Definierte Qualitätsanforderungen sollten für alle Krankenhausbereiche vorliegen.

Die AWMF und ihre Fachgesellschaften werden mit Politikern, Managern und Vertretern der Selbstverwaltung sowie Patienten bzw. Patientenvertretern Gespräche führen, um gemeinsam diese wichtigen Ziele zu erreichen.

Dieses Papier wurde unter Federführung der AWMF von der AG „Medizin und Ökonomie“ erstellt mit redaktioneller Unterstützung von Anne-Katrin Döbler (AWMF-Pressestelle).

Berlin, im Dezember 2018

\section{Interessenkonflikt}

Ina Kopp und Monika Nothacker sind Angestellte der AWMF. Sie betreuen Leitlinienprojekte methodisch und sind zuständig für die Pflege und Weiterentwicklung des AWMF-Leitlinienregisters. Peter Elsner, Manfred Gogol, Gerhard Jan Jungehülsing, Kurt Miller sowie Klaus-Peter Zimmer sind aktuell oder waren im abgefragten Zeitraum Chefärzte bzw. Klinikdirektoren. Jens Maschmann ist Medizinischer Vorstand eines Universitätsklinikums. Die weiteren Autoren erklären, dass kein Interessenkonflikt besteht.

\section{Literatur}

[1] Kreienberg R, Treede R, Wagner W. Positionen der AWMF zur Gesundheitspolitik nach der Bundestagswahl 2017- Evidenzbasierte Medizin die Basis einer guten Gesundheitspolitik 16.06.2017. Im Internet: https:// www.awmf.org/fileadmin/user_upload/Stellungnahmen/Resolution_ Forderungen/AWMF_Position_Gesundheitspolitik_lang_2017-06.pdf; Stand: 26.11.2018

[2] Nothacker M, Gaebel W, Gogol M et al. Manual - Entwicklung von Empfehlungen im Rahmen der Initiative "Gemeinsam Klug Entscheiden“ Version 1.1 von 2016. siehe „Mission“ der Ad hoc Kommission „Gemeinsam Klug Entscheiden“ 19.09.2016. Im Internet: https://www.awmf.org/ fileadmin/user_upload/Medizinische_Versorgung/GKE/Manual_GKE_ AWMF_V1-1.pdf; Stand: 26.11.2018

[3] Bundesärztekammer. (Muster-)Berufsordnung für die in Deutschland tätigen Ärztinnen und Ärzte 01.02.2019. Im Internet: http://www.bun desaerztekammer.de/fileadmin/user_upload/downloads/pdf-Ordner/ MBO/MBO-AE.pdf; Stand: 26.11.2018

[4] Zentrale Ethikkommission bei der Bundesärztekammer. Stellungnahme der Zentralen Kommission zur Wahrung ethischer Grundsätze in der Medizin und ihren Grenzgebieten - Ärztliches Handeln zwischen Berufsethos und Ökonomisierung. Das Beispiel der Verträge mit leitenden Klinikärztinnen und -ärzten. Dtsch Aerztebl 2013; 38: A1752-A1756

[5] Bundesärztekammer. Stellungnahme der Bundesärztekammer - Medizinische Indikationsstellung und Ökonomisierung. Dtsch Aerztebl 2015; 18: A836
[6] Dohmen A, Fiedler M. Ökonomisierung im Gesundheitswesen - Betriebswirtschaftlicher Erfolg als Unternehmensziel. Dtsch Aerztebl 2015; 9: A364-A366

[7] Hasenfuß G, Märker-Hermann E, Hallek M et al. INITIATIVE „KLUG ENTSCHEIDEN“ - Gegen Unter- und Überversorgung - Mitgliederbefragung zu Klug Entscheiden. Dtsch Aerztebl 2016; 13: A600 - A603

[8] Zylka-Menhorn V. Interview mit Prof. Dr. med. Gabriele Schackert, Präsidentin der Deutschen Gesellschaft für Chirurgie (DGCH) - „Chirurgie im Spannungsfeld von Technik, Ethik und Ökonomie“. Dtsch Aerztebl 2016; 16: A761 - A762

[9] Naegler H, Wehkamp KH. Medizin zwischen Patientenwohl und Ökonomisierung - Krankenhausärzte und Geschäftsführer im Interview. Berlin: Medizinisch Wissenschaftliche Verlagsgesellschaft; 2018

[10] Schumm-Draeger PM, Mann K, Mueller-Wieland D et al. Der Patient ist kein Kunde, das Krankenhaus kein Wirtschaftsunternehmen - DGIM Positionspapier warnt vor Gewinnstreben in der Klinikmedizin. Dtsch Med Wochenschr 2016; 141: 1183 -1185. doi:10.1055/s-0042-111258

[11] Bundesärztekammer. Empfehlungen der Gemeinsamen Koordinierungsstelle der Bundesärztekammer und des Verbandes der Leitenden Krankenhausärzte zu Zielvereinbarungen in Chefarztverträgen gemäß $\S 135$ c SGB V [neu]. Dtsch Aerztebl 2017; 114 (35 - 36): A1620

[12] Sachverständigenrat zur Begutachtung der Entwicklung im Gesundheitswesen (SVR Gesundheit). Gutachten 2018 - Bedarfsgerechte Steuerung der Gesundheitsversorgung. Im Internet: https:// www.svr-gesundheit.de/index.php?id=606; Stand: 26.11 .2018

[13] Van de Velde $S$, Heselmans A, Delvaux $N$ et al. A systematic review of trials evaluating success factors of interventions with computerised clinical decision support. Implementation science 2018; 13: 114

[14] Neelmeier T. Patientenaufklärung: Aufklärungspflicht als Abwehrrecht. Dtsch Aerztebl 2014; 25: A1138

[15] Zimmer KP. Wiederbelebung der Arzt-Patienten-Beziehung. Dtsch Aerztebl 2017; 42: $703-704$

[16] Deutscher Ethikrat. Patientenwohl als ethischer Maßstab für das Krankenhaus - Stellungnahme 05.04.2016. Im Internet: https://www. ethikrat.org/fileadmin/Publikationen/Stellungnahmen/deutsch/ stellungnahme-patientenwohl-als-ethischer-massstab-fuer-das-kranken haus.pdf; Stand: 26.11.2018

[17] Marckmann G, Maschmann J. Ökonomisierung: Ethische Mangelverwaltung. Dtsch Aerztebl 2017; 44: A2028

[18] Deutsche Gesellschaft für Innere Medizin (DGIM). DRG-Finanzierung der Krankenhäuser und Bonussysteme für Ärzte: Fehlentwicklungen durch falsche Anreize stoppen! 07.2012. Im Internet: https://www.dgim.de/ fileadmin/user_upload/PDF/Publikationen/Archiv/Pressemeldungen/ 2012/2012_24_Pressemitteilung.pdf; Stand: 26.11.2018

[19] Bundesinstitut für Bevölkerungsforschung. Regionaler Wandel - Regionale Herausforderungen - interaktive Karte 2015. Im Internet: https:// www.demografie-portal.de/DE/Informieren/Karte/Interaktive_Karte. html; Stand: 26.11.2018

[20] Sachverständigenrat zur Begutachtung der Entwicklung im Gesundheitswesen (SVR Gesundheit). Gutachten 2014 - Bedarfsgerechte Versorgung - Perspektiven für ländliche Regionen und ausgewählte Leistungsbereiche - Teil II: Bedarfsgerechte Versorgung aus der Perspektive ländlicher Regionen. Im Internet: https://www.svr-gesundheit.de/ index.php?id=497; Stand: 26.11 .2018

[21] Langberg EM, Dyhr L, Davidsen AS. Development of the concept of patient-centredness - A systematic review. Patient Educ Couns 2019. doi:10.1016/j.pec.2019.02.023

[22] Nowossadeck E. Demografische Alterung und Folgen für das Gesundheitssystem. GBE kompakt 2012; 3. Im Internet: https://www.rki.de/DE/ Content/Gesundheitsmonitoring/Gesundheitsberichterstattung/GBE DownloadsK/2012_2_Demografischer_Wandel_Alterung.pdf? __blob=publicationFile; Stand: 26.11.2018 
[23] Nöthen M. Hohe Kosten im Gesundheitswesen: Eine Frage des Alters? Wirtschaft und Statistik 2011; 7: 665-676

[24] Bundesministerium für Familie, Senioren, Frauen und Jugend. Übereinkommen über die Rechte des Kindes 26.01.1990. Im Internet: https://www. bmfsfj.de/blob/93140/01569e163ea92d2dd2e26b735bf59a0f/ueberein kommen-ueber-die-rechte-des-kindes-data.pdf; Stand: 26.11.2018

[25] Weyersberg A, Roth B, Woopen C. Pädiatrie: Folgen der Ökonomisierung. Dtsch Aerztebl 2018; 9: A382

[26] Kuntz B, Rattay P, Poethko-Müller C et al. Soziale Unterschiede im Gesundheitszustand von Kindern und Jugendlichen in Deutschland Querschnittergebnisse aus KiGGS Welle 2. Journal of Health Monitoring 2018; 3: 19-36. doi:10.17886/RKI-GBE-2018-076

[27] Greiner W, Batram M, Damm O et al. Kinder- und Jugendreport 2018 Gesundheitsversorgung von Kindern und Jugendlichen in Deutschland Schwerpunkt: Familiengesundheit. Im Internet: https://www.dak.de/ dak/download/dak-kinder-und-jugendreport-2018-2002322.pdf; Stand: 26.11.2108

[28] Siess M. Ärztliche Leitungsstrukturen und Führungsaufgaben - Organisationskonzepte für das moderne Krankenhaus. Wiesbaden: Dt Univ.Verl. 1999

[29] Bleß H, Kip M Hrsg. Weißbuch Gelenkersatz - Versorgungssituation endoprothetischer Hüft- und Knieoperationen in Deutschland. Berlin/ Heidelberg: Springer; 2017

[30] Arentz C, Ulrich V. Entwicklung des GKV-Beitragssatzes in mittlerer und langer Frist (2030/2060) - Mögliche Pfade und Szenarien 10/2017. Im Internet: http://www.fiwi.uni-bayreuth.de/de/download/WP_04-17. pdf; Stand: 26.11.2018

[31] Statista. Anzahl von Krankenhausbetten in OECD-Ländern in den Jahren 2015 bis 2017 (je 1000 Einwohner). Im Internet: https:// de.statista.com/statistik/daten/studie/77168/umfrage/anzahl-vonkrankenhausbetten-in-oecd-laendern/; Stand: 26.11.2018

[32] Busse R. Krankenhausstruktur weder bedarfs- noch qualitätsorientiert: zu viele Häuser, zu viele Betten, zu wenig Zentralisierung. Im Internet: https://www.mig.tu-berlin.de/fileadmin/a38331600/2018.lectures/ Magdeburg_20180418.rb_GEZEIGT.pdf; Stand: 26.11.2018

[33] Nimptsch U, Mansky T. Hospital volume and mortality for 25 types of inpatient treatment in German hospitals: observational study using complete national data from 2009 to 2014. BMJ Open 2017. doi:10.1136/bmjopen-2017-016184

[34] Statista. Durchschnittliche Verweildauer in deutschen Krankenhäusern in den Jahren 1992 bis 2017 (in Tagen). Im Internet: https://de.statista. com/statistik/daten/studie/2604/umfrage/durchschnittlicheverweildauer-im-krankenhaus-seit-1992/; Stand: 26.11.2018

[35] Statista. Prognostizierter Bedarf an Pflegekräften* in Deutschland im Jahr 2025. Im Internet: https://de.statista.com/statistik/daten/studie/ 172651/umfrage/bedarf-an-pflegekraeften-2025/; Stand: 26.11.2018

[36] Cwiertnia L, Reumschüssel A. Ärzte - Kranker Job (30.01.2016). Im Internet: https://www.zeit.de/campus/2016/01/aerzte-krankenhausgesundheit-arbeitsbedingungen-ungesund?print; Stand: 26.11.2018

[37] Koch P. Befragung junger Angestellte in Krankenhäusern - vorläufige Ergebnisse des Forschungsprojektes (05.2018). Im Internet: https://www. bgw-online.de/DE/Arbeitssicherheit-Gesundheitsschutz/GrundlagenForschung/GPR-Medientypen/Wissenschaft-Forschung/BGW08-00-043Forschungsprojekt-junge-Angestellte-Krhs.html, Stand: 26.11.2018

[38] Bundesministerium für Gesundheit. Pflegepersonaluntergrenzen (05.11.2018). Im Internet: https://www.bundesgesundheits ministerium.de/personaluntergrenzen.html; Stand: 26.11.2018

[39] Marburger Bund. Stellungnahme des Bundesverbandes zu dem Referentenentwurf des Bundesministeriums für Gesundheit 13.09.2018. Im Internet: https://www.bundesgesundheitsministerium.de/fileadmin/ Dateien/3_Downloads/Gesetze_und_Verordnungen/Stellungnahmen_ WP19/PpUGV/MB-Stellungn_PpUGV_RefEntwurf_300818_final_.pdf; Stand: 26.11 .2018
[40] Rahner E. Das Burnout-Syndrom bei Ärzten - Eine qualitative Studie zur Selbstwahrnehmung von Ursachen und Lösungsansätzen [Dissertation]. Berlin: Freie Universität Berlin. 2011

[41] Laschet H. Gesunde Ärzte, kranke Pfleger 02.04.2015. Im Internet: https://www.aerztezeitung.de/politik_gesellschaft/praevention/article/ 882907/fehlzeiten-statistik-gesunde-aerzte-kranke-pfleger.html; Stand: 6.11 .2018

[42] Braun T, Rau F, Tuschen KH. Die DRG-Einführung aus gesundheitspolitischer Sicht. Eine Zwischenbilanz. In: Klauber J, Robra BP, Schellschmidt H, Hrsg. Krankenhausreport 2007.

[43] Bundesamt für Justiz. Sozialgesetzbuch V - Gesetz zur wirtschaftlichen Sicherung der Krankenhäuser und zur Regelung der Krankenhauspflegesätze. Im Internet: http://www.gesetze-im-internet.de/khg/index. html; Stand: 26.11.2018

[44] Bundesamt für Justiz. Sozialgesetzbuch V. Gesetz über die Entgelte für voll- und teilstationäre Krankenhausleistungen. Im Internet: http:// www.gesetze-im-internet.de/khentgg/BJNR142200002.html; Stand: 26.11.2018

[45] Institut für das Entgeltsystem im Krankenhaus (gemäß §17b KH-Finanzierungsgesetz). Im Internet: https://www.g-drg.de/Das_Institut; Stand: 26.11.2018

[46] Schreyögg J. Vorschläge für eine anreizbare Reform der Krankenhausvergütung. In: Klauber J, Geraedts M, Friedrich J, et al. Hrsg. Krankenhausreport 2017 - Schwerpunkt: Zukunft gestalten. Stuttgart: Schattauer; 2017: 13-24. Im Internet: https://www.wido.de/fileadmin/ Dateien/Dokumente/Publikationen_Produkte/Buchreihen/Krankenhaus report/2017/Kapitel\%20mit\%20Deckblatt/wido_khr2017_gesamt.pdf; Stand: 26.11 .2018

[47] Busse R, Blumel M, Knieps F et al. Statutory health insurance in Germany: a health system shaped by 135 years of solidarity, self-governance, and competition. Lancet 2017; 390: $882-897$

[48] Bräutigam C, Enste P, Evans M et al. Digitalisierung im Krankenhaus Mehr Technik - Bessere Arbeit? Reihe Study der Hans-Böckler-Stiftung 2017; 364. Im Internet: https://www.boeckler.de/pdf/p_study_hbs_ 364.pdf, Stand: 26.11.2018

[49] Berger R Hrsg. Krankenhausstudie 2018. Im Internet: https://www. rolandberger.com/de/Publications/Innovationsbedarf-in-deutschenKrankenh\%C3\%A4usern.html; Stand: 03.04.2019

[50] Deutsche Krankenhausgesellschaft (DKG) Berlin. Bestandsaufnahme zur Krankenhausplanung und Investitionsfinanzierung in den Bundesländern 3/2017. Im Internet: https://www.dkgev.de/fileadmin/default/ Mediapool/1_DKG/1.7_Presse/1.7.1_Pressemitteilungen/2017/201704-18_PM-Anlage-DKG-Bestandsaufnahme_Krankenhausplanung_ Investitionsfinanzierung.pdf; Stand: 18.04.2017

[51] Deutscher Bundestag, Wissenschaftliche Dienste. Ausarbeitung Krankenhäuser in privater Trägerschaft - Rechtsgrundlagen, verfassungsrechtliche Vorgaben und Finanzierung 04.02.2014. Im Internet: https://www.bundestag.de/blob/410456/ 4e05aed207135be735046e76f13a107b/wd-9-095-13-pdf-data.pdf; Stand: 26.11 .2018

[52] Sana Kliniken AG. Geschäftsbericht 2016. Ismaning: Sana Kliniken AG; 2016

[53] Bundesamt für Justiz. Sozialgesetzbuch V - Gesetz zur Reform der Strukturen der Krankenhausversorgung (Krankenhausstrukturgesetz - KHSG). Im Internet: https://www.jurion.de/gesetze/khsg/; Stand: 26.11.2018

[54] Institut für Qualitätssicherung und Transparenz im Gesundheitswesen (IQTIG). Im Internet: https://iqtig.org/; Stand: 26.11.2018

[55] Gemeinsamer Bundesausschuss. Richtlinie zu planungsrelevanten Indikatoren 2016. Im Internet: https://www.g-ba.de/informationen/ beschluesse/2812/; Stand: 26.11.2018

[56] Institut für Qualitätssicherung und Transparenz im Gesundheitswesen (IQTIG). Qualitätsreport 2017. Im Internet: https://iqtig.org/downloads/ berichte/2017/IQTIG_Qualitaetsreport-2017_2018_09_21.pdf; Stand: 26.11.2018 